\title{
Humoral Immune Response to Selected Subgingival Plaque Microorganisms in Insulin-Dependent Diabetic Children
}

\author{
Takanobu Morinushi, ${ }^{*}$ Dennis E. Lopatin, $\dagger$ Salam A. Syed, $\dagger$ \\ George Bacon, $\ddagger$ Charles J. Kowalski, $†$ and Walter J. Loesche†
}

\begin{abstract}
JUVENILE DIABETICS HAVE BEEN SHOWN to have an increased susceptibility to gingivitis and periodontitis following puberty. However, little data are available on changes in the microbial flora that occur at the onset of puberty. This study was performed to determine if antibacterial antibody titers to selected periodontal disease-associated microorganisms might be helpful in revealing changes in plaque flora at the onset and conclusion of puberty. Sera was obtained from 35 subjects (ages 7 to 18 years) selected from a population of insulin-dependent diabetics. The subjects were given a thorough medical examination which included an assessment of sexual maturation and a dental examination which included the recording of onset and magnitude of bleeding according to the papillary bleeding score. Antibody titers to $A$. naeslundii $(A N), B$. intermedius (BI), $B$. gingivalis $(B G), F$. nucleatum $(F N), A$. actinomycetemcomitans $(A A), C$. ochracea $(C O)$ and $T$. denticola (TD) were determined using the microELISA. Stratification of antibody titers by age groups ( $\leq 12$ years, 12 to 15 years, $>15$ years) revealed that titers to AN increased significantly $(P<0.025$, ANOVA) and progressively $(P<0.05$, regression analysis) with increasing age. In contrast, the titers to $\mathrm{FN}$ were maximal in the under 12 year group and decreased with age (ANOVA, $P<$ 0.05 ; regression analysis, $P<0.05$ ). There were no significant variations in titers observed for the other microorganisms. Stratification by sexual maturity revealed a similar progressive decrease of the titer to FN (ANOVA, $P<0.05$; regression analysis, $P<0.005$ ). In addition, correlation matrix analysis at the $95 \%$ confidence level indicated that the BI titers paralleled $\mathrm{AN}$, and that the AA titer paralleled FN. While the relationship between the oral flora and diabetes is unclear, these findings suggest that there is a significant change in the oral microbial flora during puberty.
\end{abstract}

While the existence of periodontitis is rare in the prepubescent child, a change in the subgingival flora has been reported to occur concomitantly with the onset of puberty and is associated with an increased severity of gingivitis. ${ }^{1-7}$ Presumably, the establishment of a subgingival microflora more analogous to that of the adult flora establishes risk for developing periodontal disease later in life, making puberty an important period in the colonization process. The sequence of colonization leading to the establishment of the adult subgingival microbiota in children has not been well defined. However, these changes are likely to be influenced by the hormonal modulation associated with maturation, since there is evidence that the colonization

\footnotetext{
* Department of Oral Biology, School of Dentistry, The University of Michigan, Ann Arbor, MI 48109-0402.

$\dagger$ Department of Oral Biology and the Dental Research Institute.

$\ddagger$ Diabetic Research and Training Center and the Department of Pediatrics, School of Medicine.
}

of the subgingival plaque by species such as black pigmented Bacteroides species (BPB) is linked to steroid hormonal shifts in pregnant women ${ }^{8}$ and oral contraceptive use. $^{9}$

Since the study of periodontal disease in a normal circumpuberty population is difficult due to the low frequency of gingivitis, a population of insulin-dependent juvenile diabetics (IDD) was chosen for study. Previous reports have indicated that IDD have an increased level of gingivitis when compared to a nondiabetic population. ${ }^{10,11}$ As an adjunct to an ongoing study of the relationship between the subgingival microflora and puberty in this population, we decided to examine the utility of using serum antibody levels as an indicator for the presence of certain species in the subgingival plaque.

\section{MATERIALS AND METHODS}

Subjects. Thirty-five subjects (ages 7 to 18 years) were selected from a population of insulin-dependent 
diabetic (IDD) patients seen on an ongoing basis in the Department of Pediatrics, The University of Michigan Hospitals. Sera for antibody measurements were obtained from blood samples drawn for routine blood glucose determinations.

Medical and Dental Parameters. Each subject was given a thorough medical examination by the pediatric staff, which also included an assessment of sexual maturation as based on the classification proposed by Tanner and associates. ${ }^{12,13}$ Following plaque collection, a Stim-U-Dent was inserted into all interproximal sites from the midline to mesial of the first molar in all quadrants. The onset and magnitude of bleeding was recorded according to the papillary bleeding score (PBS) system. ${ }^{14}$

Plaque Collection. Interproximal plaque samples were collected in the medical examination room while the patients were sitting either in an ordinary chair or lying supine on an examining table. A small high intensity lamp was used to illuminate the field. A sterile lightning strip (Moyco) was held with a sterile forceps and inserted, with the abrasive side toward the tooth, beneath the contact point on the mesial of the upper right first molar in each subject. The strip was rubbed against the tooth surface and then dropped into a sterile vial containing $0.5 \mathrm{ml}$ of reduced transport fluid (RTF) without ethylenediaminetetraacetate. ${ }^{15}$ This sampling procedure was a modification of that previously used in a longitudinal caries investigation. ${ }^{16}$ As the strip was transferred in toto into the RTF, no microbes were lost, as could occur when small plaque samples are "shaken" off from the tip of a periodontal curette.

Microbiological Procedure. Enumeration of the plaques samples has been described previously. ${ }^{1}$ Briefly, the samples were taken into the anaerobic chamber§ within 30 to 120 minutes after collection. The plaque was vortexed for 20 seconds, after which time at $50 \mu \mathrm{l}$ aliquot was removed for counting with a darkfield microscope. The remaining suspension was diluted to $4 \mathrm{ml}$ with RTF and further dispersed for 20 seconds by sonification with a Kontes Cell Disrupter.\| The samples were serially diluted in RTF and appropriate dilutions were plated by means of a semi-automated plating devicef on the following non-selective and selective media: MM10-sucrose agar ${ }^{17}(S$. sanguis and $S$. $m u$ tans), ETSA agar ${ }^{18}$ (total count, Capnocytophaga, $F$. nucleatum, and black pigmented bacteroides), MM10 medium modified to contain $1 \%$ lactate, $0.004 \%$ bromocresol purple indicator, $5 \mu \mathrm{g} / \mathrm{ml}$ vancomycin ${ }^{19}$ (Veillonella) and ETSA agar with $2 \%$ sucrose and $20 \mu \mathrm{g}$ metronidazole ${ }^{20}$ (facultative bacteria), CFAT $\operatorname{agar}^{21}(A$. viscosus and $A$. Naeslundii), and TSBV $\operatorname{agar}^{22}$ (A. actinomycetemcomitans). In general, most colonies could

$\S$ Coy Lab Products, Ann Arbor, MI.

\| Kontes Glass Co., Vinewood, NJ.

II Spiral Systems, Inc., Cincinnati, $\mathrm{OH}$. be identified by colony morphology, however representative colonies were subcultured and additional biochemical and fermentative properties evaluated. ${ }^{23}$

Antigen Preparation. The following oral microorganisms were used as sources of antigen in this study (unless otherwise indicated, these isolates were obtained from naturally occurring gingivitis or periodontitis during the course of previous clinical studies): Actinomyces naeslundii (AN 7S, AN), Bacteroides intermedius (Forsyth strain 581,BI), Bacteroides gingivalis (JKG2, BG) Fusobacterium nucleatum (Forsyth strain 364, FN), Actinobacillus actinomycetemcomitans (Forsyth strain Y4, AA), Capnocytophaga ochracea (Forsyth strain 25, CO), and Treponema denticola (ASLM, TD). These microorganisms were grown under anaerobic conditions $\left(85 \% \mathrm{~N}_{2}, 10 \% \mathrm{H}_{2}\right.$, and $\left.5 \% \mathrm{CO}_{2}\right)$ at $37^{\circ} \mathrm{C}$ in 500 $\mathrm{ml}$ batches as previously described. ${ }^{24}$ The organisms were harvested by centrifugation at $13,000 \times \mathrm{g}$ for 20 minutes, washed three times with phosphate-buffered saline (PBS; 0.05 sodium phosphate, $0.15 \mathrm{M} \mathrm{NaCl}, \mathrm{pH}$ 7.4) containing $1 \mathrm{mM}$ EDTA. The organisms were killed and fixed by incubation with $0.5 \%$ buffered formal saline at room temperature for 16 to 18 hours on a rotary shaker. The formalinized bacteria were then washed three times with the PBS solution containing 1 mM EDTA and stored at $4^{\circ} \mathrm{C}$ in the same buffer.

Antibody Measurements. The formalinized microorganisms were diluted to an absorbance of 0.300 at 520 $\mathrm{nm}$ in $0.10 \mathrm{M}$ sodium carbonate coating buffer ( $\mathrm{pH}$ 9.6, containing $0.02 \% \mathrm{NaN}_{3}$ ). This concentration was previously determined to be optimal for sensitizing the polystyrene microtiter plates. The microtiter plates were filled $(0.2 \mathrm{ml} /$ well $)$ with the appropriate antigen preparation, incubated for 3 to 4 hours at $37^{\circ} \mathrm{C}$, and were then stored at $4^{\circ} \mathrm{C}$ until used in the assay. Before use, the wells were washed five times with PBS containing $0.05 \%$ Tween-20.* Serial dilutions of patient sera, in PBS-T, beginning with 1:64 were performed using a Titertek Medimixer. $\dagger$ A reference serum composed of a pool of at least 20 adult sera, obtained from individuals without evidence or history of periodontal disease,${ }^{24}$ possessing antibodies to all of the antigens used in the study was also titrated in each plate to allow internal standardization and calculation of ELISA units. This reference pool represented baseline adult antibody levels to the selected microorganisms. After performing the dilutions, the plates were incubated for 3 hours at room temperature. After washing five times with PBS-T a solution of alkaline phosphatasef conjugated $^{25}$ to gamma-chain specific rabbit antihuman immunoglobulin** was added to each plate $(0.1$ $\mathrm{ml} /$ well). After overnight incubation at room temper-

\footnotetext{
* PBS-T; Matheson, Coleman and Bell, Norwood, $\mathrm{OH}$.

$\dagger$ Flow Laboratories, McLean, VA.

$\ddagger$ Sigma Chemical Co., St. Louis, MO.

** Bio-Rad Laboratories, Richmond, CA.
} 
Table 1

Relationship Between Antibody Titer (IgG) and Age in Diabetic Children

\begin{tabular}{lccccc}
\hline & \multicolumn{5}{c}{ ELISA Units (Mean \pm SD) } \\
\cline { 2 - 6 } & $<12$ years & 12 to 15 years & $>15$ years & \multicolumn{2}{c}{ Statistics* } \\
\cline { 2 - 6 }$(\mathrm{n}=12)$ & $(\mathrm{n}=13)$ & $(\mathrm{n}=10)$ & ANOVA & Regr. \\
\cline { 2 - 6 } A. naeslundii & $65.9 \pm 13$ & $85.0 \pm 27$ & $135.5 \pm 71$ & $0.025 \dagger$ & 0.01 \\
B. gingivalis & $98.4 \pm 34$ & $113.3 \pm 42$ & $110.7 \pm 88$ & - & - \\
B. intermedius & $123.2 \pm 61$ & $116.7 \pm 80$ & $104.3 \pm 52$ & - & - \\
F. nucleatum & $263.5 \pm 239$ & $119.1 \pm 103$ & $89.9 \pm 24$ & $0.01 \ddagger$ & 0.05 \\
A. actinomycetemcomitans & $82.6 \pm 53$ & $68.8 \pm 29$ & $71.5 \pm 27$ & - & - \\
C. ochraceus & $118.2 \pm 55$ & $90.1 \pm 39$ & $112.2 \pm 25$ & - & - \\
T. denticola & $67.0 \pm 11$ & $71.1 \pm 31$ & $85.8 \pm 18$ & - & - \\
\hline
\end{tabular}

* Statistical analysis by univariate one-way analysis of variance and by least squares regression analysis.

$\dagger$ Significant differences between 12 to 15 year and $>15$ year groups at the $95 \%$ confidence level by Scheffe analysis.

$\ddagger$ Significant differences between $<12$ year and 12 to 15 year groups at the $95 \%$ confidence level by Scheffe analysis.

ature, the plates were again washed five times with PBST. Alkaline phosphatase substrate $\uparrow$ was added to each well. The absorbancy $(405 \mathrm{~nm})$ of each well was then determined after a development time of 30 minutes at room temperature using a multichannel spectrophotometer. $\$$ Antibody activity resident in the sera was expressed in ELISA units (EU). This value was defined by a linear regression analysis of the reference serum titration. EU of all the samples were calculated by relating optical density values from each experiment sample to the reference serum, which was assigned a value of $100 \mathrm{EU} .^{26}$ Only absorbance values occurring in the linear portion of the titration curves were used in these calculations.

Statistical Analysis. For statistical analysis, the IDD children were divided into three age groups, i.e., $<12$, 12 to 15 and $>15$ years of age and according to sexual maturity using the Tanner classification. ${ }^{12,13}$ For all comparisons, the one-way analysis of variance combined with the Scheffe test, and regression analysis were performed when appropriate. All analyses of data were performed using programs in the Michigan Interactive Data Analysis System (MIDAS).

\section{RESULTS}

Clinical Measurements. The PBS, the number of bleeding sites, and the number of sites which bled with a flow increased as a function of the age and the sexual maturation of the children as was previously reported in a larger cohort of these IDD children. ${ }^{1}$

Antibacterial Antibody Titers. Serum samples obtained from 35 children were examined for the presence of IgG antibodies to the panel of seven oral microorganisms. Serum antibodies which reacted with each member of the microbial test panel were found in all individuals. In no case could a relationship between the

$\ddagger$ Sigma Chemical Co., St. Louis, MO

† Flow Laboratories, McLean, VA. diabetic status of the subject (i.e., fasting blood glucose levels, or percent glycosylated hemoglobin) and antibody levels be determined (data not shown).

The relationships between the antibody titers and age of the child, Tanner Index, or PBS were then examined. Stratification of the antibody titers by the papillary bleeding scores (PBS) did not reveal any significant relationships (data not shown). When age was examined as the independent variable, statistically significant changes in antibody titers to AN and FN occurred. Stratification of the antibody titers into three age groups ( $<12$ years, 12 to 15 years, and $>15$ years), as shown in Table 1, revealed that humoral immunity to AN was lowest in the $<12$ and increased significantly $(P=0.025$ by ANOVA) and progressively in a linear fashion ( $P \leq$ 0.01 by regression analysis) through the other intervals. In contrast, the antibody titers to FN were maximal in the $<12$ and a significant $(P<0.05)$ and progressive decrease in titer occurred with increased age. The differences in the antibody titers to the remaining microorganisms when the various age strata were compared were not significant.

In order to determine if the sexual maturity of the patients influenced her/his antibody titers to specific microorganisms, the antibody titers were stratified according to the Tanner Index. As shown in Table 2, only FN titers demonstrated a significant decrease (ANOVA, $P \leq 0.05$; regression analysis, $P<0.005$ ) with maturity. The decrease in the FN titers paralleled the changes seen when titers were stratified by age. No significant relationships were found with the other antibody titers. We constructed a correlation matrix to determine if linkages existed between humoral antibody responses to the seven microorganisms. This might be expected to occur if they shared common antigens (cross-reaction) or because they had colonized and/or became a significant component of the flora in the gingival sulcus at the same time. The matrix, shown in Table 3, indicated that at the $P<0.05$ confidence level two relation- 
Table 2

Relationship Between Antibody Titer (IgG) and Tanner Index in Diabetic Children

\begin{tabular}{|c|c|c|c|c|c|c|c|}
\hline & \multicolumn{7}{|c|}{ ELISA Units (Mean \pm SD) } \\
\hline & \multirow{2}{*}{$\begin{array}{c}\text { Tanner } 1 \\
(n=4)\end{array}$} & \multirow{2}{*}{$\begin{array}{c}\text { Tanner } 2 \\
(\mathrm{n}=7)\end{array}$} & \multirow{2}{*}{$\begin{array}{c}\text { Tanner } 3 \\
(\mathrm{n}=8)\end{array}$} & \multirow{2}{*}{$\begin{array}{c}\text { Tanner } 4 \\
(\mathrm{n}=6)\end{array}$} & \multirow{2}{*}{$\begin{array}{l}\text { Tanner } 5 \\
(\mathrm{n}=10)\end{array}$} & \multicolumn{2}{|c|}{ Statistics* } \\
\hline & & & & & & ANOVA & Regr. \\
\hline A. naeslundii & $97.4 \pm 32$ & $79.5 \pm 21$ & $85.3 \pm 37$ & $84.6 \pm 31$ & $123.1 \pm 60$ & - & - \\
\hline B. gingivalis & $124.0 \pm 71$ & $92.1 \pm 24$ & $117.6 \pm 28$ & $101.7 \pm 62$ & $107.2 \pm 59$ & - & - \\
\hline B. intermedius & $109.6 \pm 49$ & $120.1 \pm 55$ & $132.9 \pm 103$ & $98.3 \pm 19$ & $107.6 \pm 42$ & - & - \\
\hline F. nucleatum & $254.6 \pm 213$ & $210.9 \pm 175$ & $103.5 \pm 54$ & $99.3 \pm 66$ & $86.3 \pm 21$ & $0.05 \dagger$ & 0.005 \\
\hline A. actinomycetemcomitans & $79.6 \pm 55$ & $63.3 \pm 30$ & $44.9 \pm 32$ & $55.6 \pm 8$ & $79.2 \pm 27$ & - & - \\
\hline C. ochraceus & $97.2 \pm 33$ & $92.9 \pm 13$ & $102.3 \pm 57$ & $93.9 \pm 38$ & $96.0 \pm 30$ & - & - \\
\hline$T$. denticola & $70.7 \pm 6$ & $57.1 \pm 18$ & $69.8 \pm 39$ & $77.5 \pm 41$ & $78.2 \pm 16$ & - & - \\
\hline
\end{tabular}

* Statistical analysis by univariate one-way analysis of variance and by least squares regression analysis.

$\dagger$ Significant differences between Tanner 1 to 2 and Tanner 2 to 3 groups at the $95 \%$ confidence level by Scheffe analysis.

Table 3

Relationships Between Humoral Antibody (IgG) Responses to Selected Oral Microorganisms*

\begin{tabular}{lrcccccc}
\hline \multicolumn{1}{c}{ AN } & BG & BI & FN & AA & CO & TD \\
AN & 1.0000 & & & & & & \\
BG & 0.2854 & 1.0000 & & & & & \\
BI & 0.3691 & 0.1671 & 1.0000 & & & & \\
FN & -0.1268 & 0.0331 & 0.0429 & 1.0000 & & & \\
AA & 0.0241 & 0.0157 & 0.0900 & 0.4001 & 1.0000 & & \\
CO & 0.1278 & 0.0509 & 0.0334 & 0.1001 & -0.0718 & 1.0000 & \\
TD & 0.1389 & -0.0153 & 0.0935 & -0.0106 & -0.0063 & 0.2071 & 1.0000 \\
\hline \multicolumn{7}{c}{ Correlation coefficients $(\mathrm{N}=35, \mathrm{DF}=33, \mathrm{R} @ 0.05=0.3338)}$.
\end{tabular}

ships were identified. The humoral immune response to $\mathrm{BI}$ appeared to be tied to the response to $\mathrm{AN}$, and the response to AA appeared to be related to that of FN.

Relationship Between Antibody Titers and Microbiological counts. An attempt was made to define a relationship between the microbiologic counts in the two subgingival plaque samples taken from these children with the antibody titers described above. In the cases of three of the members of the antibody panel $(B$. gingivalis, $A$. actinomycetemcomitans, and Capnocytophaga sp.), no microorganisms could be cultivated from the plaque specimens. However, the remaining four microorganisms could be detected in various concentrations in the plaque. In no instance was there a statistically significant relationship between the concentration of the microorganism in the plaque sample and its corresponding antibody titer in the serum.

\section{DISCUSSION}

We report the findings of a study which examined the antibody titers in the sera of insulin-dependent diabetic (IDD) children to a panel of subgingival plaque-associated microorganisms. Humoral immune responses to two of the microbial antigens were found to be related to age and/or sexual maturity of the individual. Increases in antibody titers to $A$. naeslundii correlated with increasing age of the child. In contrast, antibody titers to $F$. nucleatum were found to be inversely related to age. The highest titers to $\mathrm{FN}$ were found in the youngest age group. In addition, the FN titers were found to be inversely related to the level of sexual maturity of the child, i.e., the prepubescent children (under 12 years) possessed the highest antibody titers. While levels of antibacterial antibodies to the remaining members of the antigen panel could be detected in the sera of all subjects, no relationships between antibody titer and age or sexual maturity could be identified. We also attempted to determine if ties existed between antibody responses to members of the antigen panel independent of age or sexual maturity stratification. When such relationships were examined, two pairs were identified: $\mathrm{BI}$ and AN; AA and FN. The significance of these ties are not clear, but they do suggest that parameters in addition to age and sexual maturity may influence colonization and/or immune sensitization.

Our patient population was actually a subset of patients who participated in a larger study examining the relationship between puberty, diabetes, gingivitis, and plaque microbiology. In depth microbiological analysis of this larger group will be reported in a subsequent communication.

In our group of subjects, the levels of $F$. nucleatum in subgingival plaque samples were found to decrease as a function of both age and sexual maturity. This finding was recently confirmed by a report by Wojcicki et al. ${ }^{4}$ when the prepuberty and puberty groups are compared in their study. While both values (antibody titer and microbial count) suggested similar trends, attempts to demonstrate a statistically significant relationship between anti-FN antibody titer and plaque composition were unsuccessful. The level of $\mathrm{AN}$ was found to be significantly elevated in the plaque in the most immature subjects. This has also been confirmed in previous reports. ${ }^{1,2}$ Again, we found no correlation between the bacteriologic levels and the corresponding antibody titers. While the plaque levels of $B$. intermedius have been reported to increase significantly at puberty, earlier studies found no significant change in BI or other black pigmented bacteroides, ${ }^{1,3}$ confirming our findings. 
Comparisons between antibody levels and subgingival plaque flora could not be made to four of the microorganisms (TD, HA, BG, and $\mathrm{CO}$ ) since they were not detected or they were found in extremely low numbers in the plaque samples. However, only 2 of a possible 80 to 100 tooth sites were sampled so that these organisms could be present in plaques on other teeth. Spirochetes, generally found in significantly elevated levels in sites having active adult periodontal disease, ${ }^{27}$ comprised a low percentage of the microscopic count in these children. In the absence of significant levels of spirochetes, these children had an average of $75 \%$ of the normal healthy adult antibody titer to $T$. denticola [compared to the adult reference serum pool titers, where a titer of $100 \mathrm{EU}$ represents the healthy (non-periodontally-diseased) adult baseline value of our reference serum pool], indicating that while sensitization to this or a related microorganism had occurred, the antibody levels attained represented those that would normally be expected to be observed in health. Similarly, antibodies reacting with $A$ actinomycetemcomitans (AA), B. gingivalis (BG), and Capnocytophaga $s p$. were found to approximate normal adult baseline levels in the sera of these children, without significant or detectable levels in of the microorganisms in their plaque specimens. The antibodies to BG are interesting in the light that BG was rarely detected in plaque samples of these children, i.e. 2 positive isolation from over 1000 plaque samples that have been cultured (unpublished data). Other investigators have reported that BG is undetectable in most plaque samples removed from adults, thereby raising the question as to the source of $\mathrm{BG}$ antigens to which these subjects are responding. ${ }^{28}$ Titers to $\mathrm{BG}$ have been reported to increase with age and peak when certain forms of periodontitis are present. ${ }^{29}$ Capnocytophaga has been shown to elevate in gingivitis, ${ }^{30}$ but was not confirmed in our subject population. Mashimo et al., found elevated levels of serum antibody to Actinobacillus actinomycetemcomitans in five of nine juvenile (IDDM) diabetics suffering from destructive periodontitis. ${ }^{31}$ These findings were consistent with the microbiologic findings since there was little or no $B$. gingivalis found in the periodontal lesions of the juvenile diabetics, however, Actinobacillus was found in many of the lesions of the juvenile diabetics. ${ }^{31}$

The concomitant presence of antibacterial antibodies in the absence of detectable plaque species, or with a lack of correlation with the species present is confounding, but may be explained in a variety of ways. First, sensitization to certain microbial antigens may occur at low levels over a long duration. These levels may be to allow for successful detection in the plaque using classical methodology. The cumulative effect of their presence, however, may be sufficient to stimulate immunity. Also, as only two or three approximal plaques were cultured per subject, it is possible that these orga- nisms could have been present in other plaques that were not sampled. Secondly, sensitization to specific plaque microorganisms may occur as a result of short acute episodes of their prominence in the plaque. ${ }^{32}$ Once immunity has been stimulated, their presence in low, undetectable levels may be sufficient to maintain immunologic memory. Third, the antibodies in the circulation may actually be stimulated in response to cross-reactive antigens, which are unrelated to the oral status of the patient. Fourth, a reciprocal association might exist between antibody and the microbial flora, i.e., when specific antibody is elevated, the associated flora is reduced.

Finally, the lack of direct correlation between microbiologic analysis and immunity may be tied to an innate lag between the two, especially if presence of the microorganism is episodic. In studies of the effect of periodontal therapy on humoral antibody titers to selected periodontal disease associated microorganisms, it has been shown that changes in antibody titers (decreases) lagged by approximately 1 to 2 years following successful therapy. ${ }^{33,34}$ These titers probably persisted for this period of time as a result of the time required for catabolic elimination of the circulating antibodies and as a function of the maintenance of immunologic memory by undetectable levels of the organisms persisting in non-diseased, or minimally diseased sites. In this study, and in others, the instantaneous measurements made probably do not always reflect the temporal relationship between the host exposure the persistence of the immune response. This may explain the persistence and increase in the titer of AN, even though our finding, and those of others, indicates that the levels of AN are decreasing in the post-puberty group.

These factors may explain why the antibody titer may not always be expected to reflect the actual existing flora. Immunologic memory and its maintenance by minimal levels of antigen would tend to buffer episodic changes in the host flora, thus the immune response would tend to reflect the history of the antigenic load of the subject and it would be modified only with prominent and long term changes in that load.

\section{ACKNOWLEDGMENTS}

We thank Ms. Evelyn Blackburn and Mr. Neal Van Poperin for expert technical assistance. This work was supported by United States Public Health Service Grant DE 02731 from the National Institute for Dental Research.

\section{REFERENCES}

1. Gusberti, F. A., Syed, S. A., Bacon, G., Grossman, N., and Loesche, W. J.: Puberty gingivitis in insulin-dependent diabetic children. I. Cross-sectional observations. J Periodontol 54: 714, 1983.

2. Delaney, J. E., Ratzan, S. K., and Kornman, K. S.: Subgingival microbiota associated with puberty: studies of pre-, circum-, and postpubertal human females. Pediatric Dent 8: 268, 1986.

3. Yanover, L., and Ellen, R. P.: A clinical and microbiologic examination of gingival disease in parapubescent females. $J$ Perio- 
dontol 57: 562, 1986.

4. Wojcicki, C. J., Harper, D. S., and Robinson, P. J.: Differences in periodontal disease-associated subgingival plaque in prepubertal, pubertal and postpubertal children. J Periodontol 58: 219, 1987.

5. Sutcliffe, P.: A longitudinal study of gingivitis and puberty. $J$ Periodont Res 7: 52, 1972.

6. McHugh, S. E., McEwen, J. D., and Hitchin, A. D.: Dental disease and related factors in 13-year olds in Dundee. Br Dent J 117: 246, 1964.

7. Parfitt, G. J.: A five year longitudinal study of the gingival condition of a group of children in England. J Periodontol 28: 26, 1957.

8. Kornman, K. S., and Loesche, W. J.: The subgingival microbial flora during pregnancy. J Periodont Res 15: 111, 1980.

9. Jensen, J., Liljemark, W., and Bloomquist, C.: The effect of female sex hormones on subgingival plaque. $J$ Periodontol 52: 599, 1981.

10. Bernick, S. M., Cohen, D. W., Baker, L., and Laster, L.: Dental disease in children with diabetes mellitus. J Periodontol 46: $241,1975$.

11. Ringelberg, M. L., Dixon, D. O., Francis, A. O., and Plummer, R. W.: Comparison of gingival health and gingival crevicular fluid flow in children with and without diabetes. J Dent Res 56: 108, 1977.

12. Marshall, W. A., and Tanner, J. M.: Variations in the pattern of pubertal changes in girls. Arch Dis Child 44: 291, 1969.

13. Marshall, W. A., and Tanner, J. M.: Variations in the pattern of pubertal changes in boys. Arch Dis Child 45: 13, 1970.

14. Loesche, W. J.: Clinical and microbiological aspects of chemotheraputic agents used according to the specific plaque hypothesis. J Dent Res 58: 2404, 1979.

15. Syed, S. A., and Loesche, W. J.: Survival of human dental plaque flora in various transport media. Appl Microbiol 24: 638, 1972.

16. Bowden, G. H., Hardie, J. M., and Slack, G. L.: Microbial variations in approximal dental plaque. Caries Res 9: 253, 1975.

17. Loesche, W. J., and Syed, S. A.: The predominant cultivable flora of carious plaque and carious dentine. Caries Res 7: 201, 1973.

18. Syed, S. A., Svanberg, M., and Svanberg, G.: Predominant cultivable dental plaque flora of beagle dogs with gingivitis. $J$ Periodont Res 15: 123, 1980.

19. Rogosa, M.: The genus Veillonella. J Bacteriol 87: 162, 1964.

20. Loesche, W. J., Syed, S. A., Laughon, B. E., and Stoll, J.: The bacteriology of acute necrotizing ulcerative gingivitis. $J$ Periodontol 53: 233, 1982.

21. Zylber, L. J., and Jordan, H. V.: Development of a selective medium for detection and enumeration of Actinomyces viscosus and A. naeslundii in dental plaque. J Clin Microbiol 15: 253, 1982.

22. Slots, J.: Selective medium for isolation of Actinobacillus actinomycetemcomitans. J Clin Microbiol 15: 606, 1982.

23. Laughon, B. E., Syed, S. A., and Loesche, W. J.: Rapid identification of Bacteroides gingivalis. J Clin Microbiol 15: 345, 1982.

24. Lopatin, D. E., Smith, F. N., Syed, S. A., and Morrison, E. C.: The effect of periodontal therapy on lymphocyte blastogenesis to plaque associated microorganisms. J Periodont Res 18: 93, 1983.

25. Engvall, E., and Perlmann, P.: Enzyme-linked immunosorbant assay, ELISA III. Quantitation of specific antibodies by enzymelabeled anti-immunoglobulin in antigen-coated tubes. $J$ Immunol 109: 129, 1972.

26. Ebersole, J. L., Taubman, M. A., Smith, D. J., and Socransky, S. S.: Humoral immune responses and diagnosis of human periodontal disease. J Periodont Res 17: 478, 1982.

27. Loesche, W. J., Syed, S. A., Schmidt, E., and Morrison, E. C.: Bacterial profiles of subgingival plaques in periodontitis. $J$ Periodontol 56: $447,1985$.

28. Zambon, J. J., Reynolds, H. S., Chen, P., and Genco, R. J.: Rapid identification of periodontal pathogens in subgingival dental plaque. Comparison of indirect immunofluorescence microscopy with bacterial culture for detection of Bacteroides gingivalis. J Periodontal 56(11 Suppl.): 32, 1985.

29. Mouton, C., Hammond, P. G., Slots, J., and Genco, R. J.: Serum antibodies to oral Bacteroides asaccharolyticus (Bacteroides gingivalis): Relationship to age and periodontal disease. Infect Immun 31: $182,1981$.

30. Van Palenstein-Helderman, W. H.: Total viable count and differential counts of vibrio (Capnocytophaga) sputorum, Fusobacterium nucleatum, Selenomonas sputigena, Bacteroides ochraceus, and Veillonella in the inflamed and non-inflamed human gingival crevice. $J$ Periodont Res 10: 294, 1975.

31. Mashimo, P. A., Yamamoto, Y., Slots, J., Parks, B. H., and Genco, R. J.: The periodontal microflora of juvenile diabetics. Culture, immunofluorescence, and serum antibodies. $J$ Periodontol 54: $420,1983$.

32. Socransky, S. S., Haffajee, A. D., Goodson, J. M., and Linde, J.: New concepts of destructive periodontal disease. J Clin Periodontol 11: $21,1984$.

33. Aukhil, I., Lopatin, D. E., Syed, S. A., Morrison, E. C., and Kowalski, C. J.: The effects of periodontal therapy on serum antibody (IgG) levels to plaque microorganisms. J Clin Periodontol 15: 544, 1988

34. Vincent, J. W., Falkler, W. A., Cornett, W. C., and Suzuki, J. B.: Effect of periodontal therapy on specific antibody responses to suspected periodontopathogens. J Clin Periodontol 14: 412, 1987.

Send reprint requests to: Dr. Dennis E. Lopatin, 300 North Ingalls Building, Room 1192SE, School of Dentistry, The University of Michigan, Ann Arbor, Michigan 48109-0402.

Accepted for publication November 23, 1988. 